\title{
Measurement and Simulation of n-Heptane Mixture Autoignition
}

Maysam Molana ${ }^{1}$, Tusharr Goyal ${ }^{2}$, Omid Samimi-Abianeh*3

\section{Supporting Information A}

1- Experimental data at 13.5 bar

Table S1. Experimental conditions and results for mixture 1 at compressed gas pressure of 13.5 bar and high compression configuration

\begin{tabular}{|c|c|c|c|c|}
\hline $\mathrm{P}_{\mathrm{i}}$ (bar) & $\mathrm{T}_{\mathrm{i}}(\mathrm{K})$ & $\mathrm{P}_{\mathrm{c}}($ bar $)$ & $\mathrm{T}_{\mathrm{c}}(\mathrm{K})$ & $\tau(\mathrm{ms})$ \\
\hline 0.6078 & 295.46 & 13.41 & 639.55 & 16.84 \\
\hline 0.6084 & 296.04 & 13.39 & 640.39 & 17.49 \\
\hline 0.6084 & 296.04 & 13.24 & 638.70 & 15.24 \\
\hline 0.6077 & 300.00 & 13.39 & 648.13 & 10.73 \\
\hline 0.6090 & 299.95 & 13.33 & 647.05 & 11.16 \\
\hline 0.6093 & 299.93 & 13.37 & 647.30 & 10.94 \\
\hline 0.6091 & 302.37 & 13.35 & 651.78 & 8.15 \\
\hline 0.6080 & 302.35 & 13.28 & 651.28 & 8.88 \\
\hline 0.6089 & 302.28 & 13.31 & 651.30 & 8.30 \\
\hline 0.6105 & 304.97 & 13.37 & 654.32 & 6.06 \\
\hline 0.6107 & 305.15 & 13.26 & 653.34 & 7.11 \\
\hline 0.6100 & 305.10 & 13.32 & 654.14 & 7.37 \\
\hline 0.6139 & 310.82 & 13.42 & 665.57 & 4.38 \\
\hline 0.6141 & 310.49 & 13.40 & 664.62 & 5.04 \\
\hline 0.6140 & 310.01 & 13.25 & 661.94 & 4.85 \\
\hline 0.6159 & 313.19 & 13.36 & 668.79 & 4.43 \\
\hline 0.6163 & 312.89 & 13.40 & 668.62 & 4.24 \\
\hline 0.6158 & 312.98 & 13.39 & 668.78 & 4.32 \\
\hline 0.6192 & 318.93 & 13.68 & 682.42 & 2.33 \\
\hline 0.6191 & 318.60 & 13.54 & 680.33 & 2.59 \\
\hline 0.6189 & 318.28 & 13.52 & 679.45 & 2.17 \\
\hline 0.6190 & 323.57 & 13.67 & 691.16 & 1.49 \\
\hline 0.6186 & 323.18 & 13.56 & 689.41 & 1.37 \\
\hline 0.6190 & 322.89 & 13.47 & 687.39 & 1.56 \\
\hline 0.6199 & 328.03 & 13.65 & 698.83 & 1.18 \\
\hline 0.6198 & 327.64 & 13.54 & 697.03 & 1.14 \\
\hline 0.6195 & 327.72 & 13.39 & 697.91 & 1.45 \\
\hline 0.6213 & 338.07 & 13.34 & 713.42 & 0.94 \\
\hline 0.6211 & 339.00 & 13.12 & 712.76 & 1.10 \\
\hline & & & & \\
\hline
\end{tabular}

\footnotetext{
${ }^{1} \mathrm{PhD}$ student, Mechanical Engineering Department, Wayne State University, Detroit, MI, United States, molana@wayne.edu

${ }^{2} \mathrm{PhD}$ student, Mechanical Engineering Department, Wayne State University, Detroit, MI, United States, tusharrgoyel@wayne.edu

${ }^{3}$ Corresponding author, Assistant professor, Wayne State University, Mechanical Engineering Department, Detroit, MI, United States, O.Samimi@wayne.edu
} 


$$
\begin{array}{|l|l|l|l|l|}
0.6213 & 338.70 & 13.43 & 715.69 & 0.94 \\
\hline
\end{array}
$$

Table S2. Experimental conditions and results for mixture 4 (inert) at compressed gas pressure of 13.5 bar and high compression configuration

\begin{tabular}{|c|c|c|c|}
\hline $\mathrm{P}_{\mathrm{i}}($ bar $)$ & $\mathrm{T}_{\mathrm{i}}(\mathrm{K})$ & $\mathrm{P}_{\mathrm{c}}($ bar $)$ & $\mathrm{T}_{\mathrm{c}}(\mathrm{K})$ \\
\hline 0.6071 & 297.18 & 13.43 & 644.28 \\
\hline 0.6074 & 297.18 & 13.50 & 645.00 \\
\hline 0.6076 & 299.80 & 13.54 & 650.61 \\
\hline 0.6073 & 299.72 & 13.40 & 649.03 \\
\hline 0.6082 & 302.87 & 13.45 & 655.27 \\
\hline 0.6090 & 302.76 & 13.37 & 660.20 \\
\hline 0.6092 & 305.91 & 13.39 & 660.20 \\
\hline 0.6091 & 305.45 & 13.36 & 658.99 \\
\hline 0.6089 & 308.15 & 13.31 & 663.63 \\
\hline 0.6089 & 307.55 & 13.29 & 662.52 \\
\hline 0.6160 & 313.91 & 13.56 & 672.25 \\
\hline 0.6163 & 313.47 & 13.47 & 670.38 \\
\hline 0.6194 & 318.98 & 13.46 & 679.99 \\
\hline 0.6192 & 318.91 & 13.43 & 679.49 \\
\hline 0.6189 & 323.74 & 13.42 & 688.56 \\
\hline 0.6191 & 323.53 & 13.42 & 688.18 \\
\hline 0.6192 & 327.78 & 13.42 & 695.82 \\
\hline 0.6183 & 327.79 & 13.35 & 695.46 \\
\hline 0.6212 & 333.52 & 13.37 & 705.70 \\
\hline 0.6218 & 332.68 & 13.39 & 704.20 \\
\hline
\end{tabular}


Table S3. Experimental conditions and results for mixture 1 at compressed gas pressure of 13.5 bar and medium compression configuration

\begin{tabular}{|c|c|c|c|c|}
\hline $\mathrm{P}_{\mathrm{i}}$ (bar) & $\mathrm{T}_{\mathrm{i}}(\mathrm{K})$ & $\mathrm{P}_{\mathrm{c}}($ bar $)$ & $\mathrm{T}_{\mathrm{c}}(\mathrm{K})$ & $\tau(\mathrm{ms})$ \\
\hline 0.7537 & 299.15 & 13.47 & 613.53 & 49.23 \\
\hline 0.7538 & 299.18 & 13.61 & 614.84 & 47.32 \\
\hline 0.7532 & 299.19 & 13.60 & 615.05 & 45.87 \\
\hline 0.7529 & 297.03 & 13.49 & 609.91 & 68.70 \\
\hline 0.7522 & 297.10 & 13.47 & 609.89 & 71.32 \\
\hline 0.7520 & 297.11 & 13.50 & 610.34 & 68.97 \\
\hline 0.7513 & 302.35 & 13.25 & 618.00 & 47.73 \\
\hline 0.7523 & 302.25 & 13.39 & 618.59 & 44.01 \\
\hline 0.7523 & 302.21 & 13.35 & 618.10 & 45.54 \\
\hline 0.7524 & 305.53 & 13.31 & 623.52 & 30.87 \\
\hline 0.7525 & 305.42 & 13.33 & 623.76 & 31.05 \\
\hline 0.7524 & 305.25 & 13.31 & 623.34 & 31.90 \\
\hline 0.7517 & 308.37 & 13.32 & 629.38 & 25.49 \\
\hline 0.7523 & 308.24 & 13.29 & 628.96 & 24.95 \\
\hline 0.7526 & 307.99 & 13.29 & 627.86 & 23.77 \\
\hline 0.7527 & 310.83 & 13.27 & 632.84 & 19.15 \\
\hline 0.7524 & 310.88 & 13.27 & 632.94 & 19.37 \\
\hline 0.7522 & 310.90 & 13.26 & 632.96 & 19.91 \\
\hline 0.7534 & 313.93 & 13.21 & 637.83 & 14.99 \\
\hline 0.7540 & 313.86 & 13.28 & 638.15 & 13.82 \\
\hline 0.7533 & 313.83 & 13.21 & 637.67 & 14.84 \\
\hline 0.7559 & 316.73 & 13.26 & 642.73 & 10.73 \\
\hline 0.7559 & 316.64 & 13.25 & 642.57 & 11.09 \\
\hline 0.7557 & 316.63 & 13.22 & 642.12 & 11.22 \\
\hline 0.7562 & 319.62 & 13.21 & 647.32 & 9.18 \\
\hline 0.7557 & 319.37 & 13.21 & 646.89 & 10.36 \\
\hline 0.7552 & 319.43 & 13.18 & 646.99 & 9.20 \\
\hline 0.7581 & 322.31 & 13.27 & 652.44 & 7.01 \\
\hline 0.7584 & 322.09 & 13.23 & 651.61 & 6.73 \\
\hline 0.7581 & 322.60 & 13.17 & 652.06 & 7.83 \\
\hline
\end{tabular}


Table S4. Experimental conditions and results for mixture 4 (inert) at compressed gas pressure of 13.5 bar and medium compression configuration

\begin{tabular}{|c|c|c|c|}
\hline $\mathrm{P}_{\mathrm{i}}($ bar $)$ & $\mathrm{T}_{\mathrm{i}}(\mathrm{K})$ & $\mathrm{P}_{\mathrm{c}}($ bar $)$ & $\mathrm{T}_{\mathrm{c}}(\mathrm{K})$ \\
\hline 0.7531 & 300.04 & 13.67 & 620.20 \\
\hline 0.7529 & 299.89 & 13.64 & 619.80 \\
\hline 0.7523 & 303.55 & 13.61 & 626.40 \\
\hline 0.7523 & 303.51 & 13.61 & 626.34 \\
\hline 0.7522 & 305.70 & 13.61 & 630.26 \\
\hline 0.7522 & 305.38 & 13.56 & 629.27 \\
\hline 0.7523 & 308.85 & 13.58 & 635.93 \\
\hline 0.7516 & 308.39 & 13.53 & 634.66 \\
\hline 0.7520 & 310.89 & 13.50 & 638.71 \\
\hline 0.7523 & 311.09 & 13.49 & 639.07 \\
\hline 0.7534 & 314.90 & 13.45 & 645.65 \\
\hline 0.7530 & 314.14 & 13.46 & 644.30 \\
\hline 0.7531 & 317.25 & 13.50 & 650.44 \\
\hline 0.7526 & 316.93 & 13.44 & 649.27 \\
\hline 0.7530 & 321.00 & 13.41 & 656.49 \\
\hline 0.7534 & 319.70 & 13.43 & 654.19 \\
\hline 0.7574 & 322.66 & 13.50 & 659.74 \\
\hline 0.7586 & 322.86 & 13.55 & 660.41 \\
\hline
\end{tabular}


Table S5. Experimental conditions and results for mixture 2 at compressed gas pressure of 13.5 bar and low compression configuration

\begin{tabular}{|c|c|c|c|c|}
\hline $\mathrm{P}_{\mathrm{i}}(\mathrm{bar})$ & $\mathrm{T}_{\mathrm{i}}(\mathrm{K})$ & $\mathrm{P}_{\mathrm{c}}(\mathrm{bar})$ & $\mathrm{T}_{\mathrm{c}}(\mathrm{K})$ & $\tau(\mathrm{ms})$ \\
\hline 0.7996 & 287.54 & 12.91 & 604.75 & 93.98 \\
\hline 0.8046 & 288.01 & 12.93 & 605.18 & 89.37 \\
\hline 0.8039 & 288.15 & 12.91 & 605.42 & 91.14 \\
\hline 0.8110 & 291.29 & 12.95 & 610.21 & 55.04 \\
\hline 0.8109 & 291.40 & 12.93 & 610.40 & 57.19 \\
\hline 0.81083 & 291.47 & 12.93 & 610.53 & 59.59 \\
\hline 0.8398 & 294.87 & 13.35 & 616.20 & 47.38 \\
\hline 0.8400 & 294.31 & 13.34 & 615.19 & 46.84 \\
\hline 0.8396 & 294.20 & 13.31 & 614.55 & 45.54 \\
\hline 0.8439 & 296.65 & 13.39 & 619.40 & 34.83 \\
\hline 0.8442 & 296.25 & 13.42 & 618.92 & 36.11 \\
\hline 0.8441 & 296.16 & 13.39 & 618.52 & 37.86 \\
\hline 0.8464 & 298.98 & 13.38 & 623.12 & 28.24 \\
\hline 0.8461 & 298.87 & 13.39 & 622.92 & 28.87 \\
\hline 0.8463 & 298.94 & 13.32 & 622.38 & 30.51 \\
\hline 0.8496 & 301.89 & 13.40 & 628.31 & 18.39 \\
\hline 0.8499 & 302.75 & 13.37 & 629.40 & 19.44 \\
\hline 0.8503 & 302.32 & 13.39 & 628.64 & 20.85 \\
\hline 0.8528 & 304.39 & 13.37 & 631.87 & 17.01 \\
\hline 0.8538 & 304.41 & 13.32 & 630.98 & 18.96 \\
\hline 0.8529 & 304.46 & 13.31 & 631.07 & 17.92 \\
\hline 0.8571 & 307.92 & 13.39 & 637.69 & 12.40 \\
\hline 0.8575 & 307.65 & 13.35 & 636.74 & 14.25 \\
\hline 0.8575 & 307.61 & 13.37 & 637.13 & 13.81 \\
\hline 0.8607 & 310.79 & 13.38 & 642.30 & 9.88 \\
\hline 0.8601 & 310.69 & 13.31 & 641.40 & 11.44 \\
\hline 0.8455 & 310.76 & 13.11 & 642.24 & 11.40 \\
\hline 0.8662 & 313.77 & 13.64 & 649.89 & 5.32 \\
\hline 0.8665 & 313.19 & 13.53 & 647.48 & 6.93 \\
\hline 0.8664 & 312.84 & 13.51 & 646.60 & 7.14 \\
\hline 0.8661 & 316.81 & 13.39 & 652.47 & 7.31 \\
\hline 0.8658 & 316.84 & 13.39 & 652.52 & 8.00 \\
\hline 0.8655 & 316.78 & 13.39 & 653.10 & 7.98 \\
\hline 0.8667 & 320.21 & 13.34 & 657.97 & 7.17 \\
\hline 0.8658 & 319.70 & 13.32 & 657.08 & 6.22 \\
\hline 0.8668 & 319.60 & 13.34 & 656.90 & 6.58 \\
\hline 0.8662 & 322.77 & 13.40 & 663.41 & 4.04 \\
\hline 0.8661 & 322.74 & 13.35 & 662.88 & 5.28 \\
\hline 0.8661 & 322.67 & 13.36 & 662.76 & 5.30 \\
\hline 0.8669 & 326.65 & 13.44 & 669.16 & 4.85 \\
\hline 0.8670 & 326.79 & 13.30 & 668.12 & 4.06 \\
\hline 0.8667 & 325.82 & 13.23 & 665.10 & 4.78 \\
\hline 0.8673 & 329.11 & 13.26 & 671.36 & 3.08 \\
\hline 0.8673 & 329.25 & 13.24 & 671.32 & 3.63 \\
\hline 0.8673 & 328.91 & 13.26 & 671.01 & 3.72 \\
\hline 0.8671 & 331.47 & 13.26 & 675.71 & 2.71 \\
\hline 0.8674 & 331.58 & 13.26 & 675.75 & 2.53 \\
\hline 0.8676 & 331.59 & 13.23 & 675.41 & 2.78 \\
\hline & & & & \\
\hline
\end{tabular}


Table S6. Experimental conditions and results for mixture 5 (inert) at compressed gas pressure of 13.5 bar and low compression configuration

\begin{tabular}{|c|c|c|c|}
\hline $\mathrm{P}_{\mathrm{i}}($ bar $)$ & $\mathrm{T}_{\mathrm{i}}(\mathrm{K})$ & $\mathrm{P}_{\mathrm{c}}($ bar $)$ & $\mathrm{T}_{\mathrm{c}}(\mathrm{K})$ \\
\hline 0.8105 & 292.64 & 12.88 & 614.76 \\
\hline 0.8100 & 292.72 & 12.90 & 615.32 \\
\hline 0.8438 & 297.09 & 13.45 & 623.70 \\
\hline 0.8447 & 296.95 & 13.38 & 622.64 \\
\hline 0.8451 & 299.52 & 13.45 & 628.13 \\
\hline 0.8453 & 299.44 & 13.41 & 627.59 \\
\hline 0.8479 & 302.93 & 13.41 & 633.54 \\
\hline 0.8476 & 302.94 & 13.39 & 633.55 \\
\hline 0.8521 & 306.05 & 13.53 & 638.47 \\
\hline 0.8538 & 305.83 & 13.47 & 636.84 \\
\hline 0.8564 & 309.12 & 13.52 & 643.19 \\
\hline 0.8566 & 308.75 & 13.44 & 641.70 \\
\hline 0.8605 & 311.71 & 13.68 & 649.12 \\
\hline 0.8601 & 311.26 & 13.53 & 646.63 \\
\hline 0.8667 & 313.99 & 13.53 & 650.69 \\
\hline 0.8658 & 314.34 & 13.45 & 650.48 \\
\hline 0.8663 & 316.86 & 13.62 & 657.11 \\
\hline 0.8660 & 316.91 & 13.60 & 656.78 \\
\hline 0.8657 & 319.97 & 13.48 & 661.40 \\
\hline 0.8663 & 320.92 & 13.51 & 663.10 \\
\hline 0.8659 & 323.06 & 13.53 & 667.52 \\
\hline 0.8661 & 323.91 & 13.45 & 668.01 \\
\hline 0.8667 & 326.35 & 13.45 & 672.34 \\
\hline 0.8663 & 326.93 & 13.45 & 673.38 \\
\hline 0.8673 & 328.73 & 13.75 & 680.48 \\
\hline 0.8672 & 328.73 & 13.58 & 678.32 \\
\hline 0.8670 & 332.24 & 13.55 & 684.55 \\
\hline 0.8670 & 333.02 & 13.44 & 684.63 \\
\hline 0.8669 & 334.98 & 13.39 & 687.65 \\
\hline 0.8666 & 334.63 & 13.42 & 687.48 \\
\hline
\end{tabular}


Table S7. Experimental conditions and results for mixture 3 at compressed gas pressure of 13.5 bar and high compression configuration

\begin{tabular}{|c|c|c|c|c|c|}
\hline $\mathrm{P}_{\mathrm{i}}(\mathrm{bar})$ & $\mathrm{T}_{\mathrm{i}}(\mathrm{K})$ & $\mathrm{P}_{\mathrm{c}}(\mathrm{bar})$ & $\mathrm{T}_{\mathrm{c}}(\mathrm{K})$ & $\tau(\mathrm{ms})$ & $\begin{array}{c}\text { First stage ignition } \\
\text { delay }(\mathrm{ms})\end{array}$ \\
\hline 0.5739 & 297.84 & 13.68 & 667.88 & 12.30 & 5.77 \\
\hline 0.5739 & 297.96 & 13.62 & 667.43 & 12.01 & 5.90 \\
\hline 0.5733 & 298.03 & 13.60 & 667.57 & 11.60 & 5.85 \\
\hline 0.5703 & 304.01 & 13.44 & 678.37 & 9.28 & 3.96 \\
\hline 0.5710 & 303.97 & 13.45 & 678.29 & 9.06 & 3.82 \\
\hline 0.5714 & 303.89 & 13.50 & 678.49 & 9.02 & 3.82 \\
\hline 0.5733 & 308.37 & 13.41 & 686.00 & 6.64 & 2.81 \\
\hline 0.5734 & 308.12 & 13.46 & 685.88 & 7.05 & 2.56 \\
\hline 0.5737 & 307.98 & 13.47 & 685.60 & 6.72 & 2.63 \\
\hline 0.5868 & 312.96 & 13.72 & 694.74 & 5.96 & 1.61 \\
\hline 0.5836 & 312.90 & 13.64 & 694.63 & 6.65 & 1.44 \\
\hline 0.5795 & 312.97 & 13.55 & 694.75 & 6.31 & 1.71 \\
\hline 0.5793 & 318.85 & 13.52 & 706.28 & 5.38 & 1.07 \\
\hline 0.5792 & 318.29 & 13.47 & 704.49 & 5.36 & 0.98 \\
\hline 0.5794 & 318.05 & 13.47 & 704.04 & 4.98 & 1.07 \\
\hline 0.5828 & 322.89 & 13.66 & 715.00 & 5.17 & 0.51 \\
\hline 0.5827 & 322.86 & 13.64 & 714.58 & 4.51 & 0.46 \\
\hline 0.5825 & 322.81 & 13.66 & 714.85 & 4.59 & 0.59 \\
\hline
\end{tabular}


Table S8. Experimental conditions and results for mixture 6 (inert) at compressed gas pressure of 13.5 bar and high compression configuration

\begin{tabular}{|c|c|c|c|}
\hline $\mathrm{P}_{\mathrm{i}}($ bar $)$ & $\mathrm{T}_{\mathrm{i}}(\mathrm{K})$ & $\mathrm{P}_{\mathrm{c}}($ bar $)$ & $\mathrm{T}_{\mathrm{c}}(\mathrm{K})$ \\
\hline 0.5958 & 295.98 & 14.10 & 665.68 \\
\hline 0.5965 & 296.03 & 14.02 & 664.70 \\
\hline 0.5902 & 304.03 & 13.76 & 679.92 \\
\hline 0.5905 & 303.80 & 13.73 & 678.74 \\
\hline 0.5884 & 308.94 & 13.60 & 687.93 \\
\hline 0.5886 & 308.64 & 13.57 & 687.35 \\
\hline 0.5909 & 313.32 & 13.55 & 695.61 \\
\hline 0.5900 & 313.78 & 13.49 & 696.11 \\
\hline 0.5906 & 317.91 & 13.53 & 704.42 \\
\hline 0.5895 & 317.93 & 13.84 & 708.61 \\
\hline 0.5909 & 322.83 & 13.99 & 719.87 \\
\hline 0.5893 & 322.89 & 13.67 & 716.64 \\
\hline 0.5904 & 328.97 & 13.79 & 729.76 \\
\hline 0.5911 & 328.90 & 13.66 & 727.71 \\
\hline 0.5888 & 332.68 & 13.62 & 735.68 \\
\hline 0.5880 & 332.79 & 13.26 & 731.55 \\
\hline 0.5898 & 337.75 & 13.65 & 745.29 \\
\hline 0.5904 & 337.72 & 13.66 & 745.62 \\
\hline 0.5899 & 342.12 & 13.72 & 755.08 \\
\hline 0.5905 & 342.89 & 13.77 & 756.92 \\
\hline
\end{tabular}




\section{2- Experimental data at 6.5 bar}

Table S9. Experimental conditions and results for mixture 1 at compressed gas pressure of 6.5 bar and high compression configuration

\begin{tabular}{|c|c|c|c|c|}
\hline $\mathrm{P}_{\mathrm{i}}($ bar $)$ & $\mathrm{T}_{\mathrm{i}}(\mathrm{K})$ & $\mathrm{P}_{\mathrm{c}}(\mathrm{bar})$ & $\mathrm{T}_{\mathrm{c}}(\mathrm{K})$ & $\tau(\mathrm{ms})$ \\
\hline 0.3010 & 305.07 & 6.43 & 650.77 & 12.64 \\
\hline 0.3015 & 305.09 & 6.45 & 651.06 & 13.60 \\
\hline 0.3014 & 305.12 & 6.41 & 650.30 & 13.92 \\
\hline 0.3022 & 292.66 & 6.54 & 629.12 & 47.37 \\
\hline 0.3019 & 292.88 & 6.54 & 629.80 & 46.23 \\
\hline 0.3017 & 293.60 & 6.48 & 629.82 & 45.09 \\
\hline 0.3015 & 297.31 & 6.53 & 638.18 & 26.96 \\
\hline 0.3012 & 297.24 & 6.49 & 637.28 & 28.53 \\
\hline 0.3013 & 297.20 & 6.52 & 637.72 & 27.90 \\
\hline 0.3019 & 299.70 & 6.55 & 643.07 & 22.30 \\
\hline 0.3014 & 299.49 & 6.49 & 641.39 & 22.81 \\
\hline 0.3012 & 299.52 & 6.46 & 640.93 & 24.10 \\
\hline 0.3017 & 302.96 & 6.48 & 647.72 & 18.01 \\
\hline 0.3017 & 302.95 & 6.43 & 646.37 & 20.23 \\
\hline 0.3013 & 302.78 & 6.41 & 645.79 & 19.00 \\
\hline 0.3030 & 307.42 & 6.49 & 655.56 & 14.20 \\
\hline 0.3030 & 307.55 & 6.43 & 654.45 & 13.52 \\
\hline 0.3026 & 307.59 & 6.31 & 651.96 & 12.50 \\
\hline 0.3027 & 310.38 & 6.45 & 660.38 & 11.62 \\
\hline 0.3026 & 310.39 & 6.42 & 659.85 & 12.01 \\
\hline 0.3022 & 310.40 & 6.37 & 658.76 & 11.68 \\
\hline 0.3027 & 313.05 & 6.34 & 662.87 & 9.85 \\
\hline 0.3027 & 313.15 & 6.37 & 663.73 & 10.87 \\
\hline 0.3021 & 313.18 & 6.39 & 664.62 & 9.76 \\
\hline 0.3023 & 317.53 & 6.40 & 673.02 & 10.27 \\
\hline 0.3020 & 317.54 & 6.41 & 673.31 & 10.10 \\
\hline 0.3023 & 316.95 & 6.43 & 672.53 & 10.04 \\
\hline & & & & \\
\hline
\end{tabular}


Table S10. Experimental conditions and results for mixture 4 (inert) at compressed gas pressure of 6.5 bar and high compression configuration

\begin{tabular}{|c|c|c|c|}
\hline $\mathrm{P}_{\mathrm{i}}($ bar $)$ & $\mathrm{T}_{\mathrm{i}}(\mathrm{K})$ & $\mathrm{P}_{\mathrm{c}}($ bar $)$ & $\mathrm{T}_{\mathrm{c}}(\mathrm{K})$ \\
\hline 0.7531 & 300.04 & 13.67 & 620.20 \\
\hline 0.7529 & 299.89 & 13.64 & 619.80 \\
\hline 0.7523 & 303.55 & 13.61 & 626.40 \\
\hline 0.7523 & 303.51 & 13.61 & 626.34 \\
\hline 0.7522 & 305.70 & 13.61 & 630.26 \\
\hline 0.7522 & 305.38 & 13.56 & 629.27 \\
\hline 0.7523 & 308.85 & 13.58 & 635.93 \\
\hline 0.7516 & 308.39 & 13.53 & 634.66 \\
\hline 0.7520 & 310.89 & 13.50 & 638.71 \\
\hline 0.7523 & 311.09 & 13.49 & 639.07 \\
\hline 0.7534 & 314.90 & 13.45 & 645.65 \\
\hline 0.7530 & 314.14 & 13.46 & 644.30 \\
\hline 0.7531 & 317.25 & 13.50 & 650.44 \\
\hline 0.7526 & 316.93 & 13.44 & 649.27 \\
\hline 0.7530 & 321.00 & 13.41 & 656.49 \\
\hline 0.7534 & 319.70 & 13.43 & 654.19 \\
\hline 0.7574 & 322.66 & 13.50 & 659.74 \\
\hline 0.7586 & 322.86 & 13.55 & 660.41 \\
\hline
\end{tabular}


Table S11. Experimental conditions and results for mixture 1 at compressed gas pressure of 6.5 bar and medium compression configuration

\begin{tabular}{|c|c|c|c|c|}
\hline $\mathrm{P}_{\mathrm{i}}(\mathrm{bar})$ & $\mathrm{T}_{\mathrm{i}}(\mathrm{K})$ & $\mathrm{P}_{\mathrm{c}}(\mathrm{bar})$ & $\mathrm{T}_{\mathrm{c}}(\mathrm{K})$ & $\tau(\mathrm{ms})$ \\
\hline 0.3938 & 299.21 & 6.64 & 605.18 & 84.50 \\
\hline 0.3940 & 299.13 & 6.82 & 608.86 & 80.95 \\
\hline 0.3939 & 299.13 & 6.73 & 607.16 & 78.89 \\
\hline 0.3937 & 302.18 & 6.84 & 615.08 & 66.26 \\
\hline 0.3940 & 302.07 & 6.86 & 614.89 & 66.27 \\
\hline 0.3942 & 302.03 & 6.85 & 614.82 & 66.05 \\
\hline 0.3949 & 305.01 & 6.85 & 620.06 & 51.90 \\
\hline 0.3906 & 305.01 & 6.85 & 621.33 & 51.27 \\
\hline 0.3931 & 304.97 & 6.83 & 619.99 & 51.13 \\
\hline 0.3926 & 308.00 & 6.80 & 624.87 & 39.18 \\
\hline 0.3927 & 307.92 & 6.81 & 625.17 & 38.71 \\
\hline 0.3919 & 307.95 & 6.82 & 626.00 & 36.54 \\
\hline 0.3910 & 311.24 & 6.79 & 631.41 & 28.05 \\
\hline 0.3908 & 310.77 & 6.77 & 630.14 & 28.77 \\
\hline 0.3907 & 310.79 & 6.78 & 630.62 & 29.72 \\
\hline 0.3910 & 313.64 & 6.75 & 634.73 & 23.28 \\
\hline 0.3908 & 313.55 & 6.74 & 634.56 & 22.98 \\
\hline 0.3909 & 313.77 & 6.74 & 634.08 & 21.62 \\
\hline 0.3907 & 316.62 & 6.71 & 639.48 & 18.70 \\
\hline 0.3899 & 316.64 & 6.74 & 640.38 & 19.12 \\
\hline 0.3905 & 316.64 & 6.70 & 639.50 & 17.08 \\
\hline 0.3903 & 320.86 & 6.70 & 647.28 & 14.52 \\
\hline 0.3915 & 320.75 & 6.69 & 646.21 & 14.66 \\
\hline 0.3911 & 319.99 & 6.69 & 644.89 & 15.34 \\
\hline 0.3908 & 322.78 & 6.67 & 649.72 & 12.44 \\
\hline 0.3899 & 322.63 & 6.57 & 647.24 & 12.51 \\
\hline 0.3898 & 322.66 & 6.66 & 649.50 & 12.97 \\
\hline 0.3899 & 325.42 & 6.61 & 653.39 & 9.55 \\
\hline 0.3894 & 325.55 & 6.61 & 653.60 & 9.82 \\
\hline 0.3902 & 325.65 & 6.62 & 653.79 & 9.91 \\
\hline 0.3896 & 329.61 & 6.59 & 660.15 & 8.48 \\
\hline 0.3892 & 328.58 & 6.60 & 658.83 & 8.48 \\
\hline 0.3887 & 328.54 & 6.55 & 657.86 & 9.50 \\
\hline & & & & \\
\hline
\end{tabular}


Table S12. Experimental conditions and results for mixture 4 (inert) at compressed gas pressure of 6.5 bar and medium compression configuration

\begin{tabular}{|c|c|c|c|}
\hline $\mathrm{P}_{\mathrm{i}}($ bar $)$ & $\mathrm{T}_{\mathrm{i}}(\mathrm{K})$ & $\mathrm{P}_{\mathrm{c}}(\mathrm{bar})$ & $\mathrm{T}_{\mathrm{c}}(\mathrm{K})$ \\
\hline 0.3932 & 299.71 & 6.99 & 616.92 \\
\hline 0.3934 & 299.64 & 6.97 & 615.93 \\
\hline 0.3923 & 303.11 & 6.96 & 622.60 \\
\hline 0.3929 & 303.48 & 6.96 & 622.84 \\
\hline 0.3928 & 305.23 & 6.96 & 626.39 \\
\hline 0.3926 & 305.08 & 6.94 & 625.69 \\
\hline 0.3961 & 308.12 & 6.97 & 630.70 \\
\hline 0.3922 & 307.83 & 6.90 & 630.19 \\
\hline 0.3904 & 311.82 & 6.90 & 637.73 \\
\hline 0.3902 & 310.80 & 6.87 & 635.92 \\
\hline 0.3904 & 313.68 & 6.86 & 641.02 \\
\hline 0.3899 & 313.41 & 6.85 & 640.53 \\
\hline 0.3903 & 316.74 & 6.81 & 645.51 \\
\hline 0.3899 & 316.68 & 6.82 & 645.72 \\
\hline 0.3900 & 319.81 & 6.80 & 650.94 \\
\hline 0.3895 & 319.36 & 6.81 & 650.46 \\
\hline 0.3900 & 322.82 & 6.82 & 656.90 \\
\hline 0.3887 & 322.51 & 6.79 & 656.34 \\
\hline 0.3904 & 326.80 & 6.77 & 662.95 \\
\hline 0.3886 & 326.12 & 6.76 & 662.06 \\
\hline 0.3904 & 328.53 & 6.75 & 665.65 \\
\hline 0.3984 & 327.83 & 6.89 & 664.42 \\
\hline 0.3901 & 331.65 & 6.73 & 671.13 \\
\hline 0.3900 & 331.22 & 6.72 & 670.05 \\
\hline
\end{tabular}


Table S13. Experimental conditions and results for mixture 2 at compressed gas pressure of 6.5 bar and low compression configuration

\begin{tabular}{|c|c|c|c|c|}
\hline $\mathrm{P}_{\mathrm{i}}(\mathrm{bar})$ & $\mathrm{T}_{\mathrm{i}}(\mathrm{K})$ & $\mathrm{P}_{\mathrm{c}}(\mathrm{bar})$ & $\mathrm{T}_{\mathrm{c}}(\mathrm{K})$ & $\tau(\mathrm{ms})$ \\
\hline 0.4136 & 296.12 & 6.15 & 608.57 & 78.79 \\
\hline 0.4145 & 296.55 & 6.21 & 610.23 & 77.59 \\
\hline 0.4140 & 296.21 & 6.20 & 609.62 & 78.20 \\
\hline 0.4152 & 299.66 & 6.38 & 619.83 & 59.40 \\
\hline 0.4157 & 299.30 & 6.40 & 619.63 & 59.18 \\
\hline 0.4157 & 299.04 & 6.41 & 619.62 & 61.86 \\
\hline 0.4202 & 301.95 & 6.47 & 624.81 & 35.51 \\
\hline 0.4211 & 301.83 & 6.48 & 624.14 & 36.53 \\
\hline 0.4219 & 301.94 & 6.48 & 624.34 & 35.09 \\
\hline 0.4316 & 304.49 & 6.61 & 628.40 & 28.86 \\
\hline 0.4321 & 304.44 & 6.60 & 627.86 & 29.68 \\
\hline 0.4318 & 304.43 & 6.61 & 628.30 & 29.03 \\
\hline 0.4355 & 307.58 & 6.61 & 632.49 & 24.12 \\
\hline 0.4363 & 307.49 & 6.63 & 632.79 & 23.26 \\
\hline 0.4356 & 307.45 & 6.63 & 633.17 & 23.22 \\
\hline 0.4371 & 310.91 & 6.60 & 637.88 & 17.82 \\
\hline 0.4368 & 312.02 & 6.60 & 639.85 & 17.45 \\
\hline 0.4366 & 311.53 & 6.59 & 638.98 & 17.81 \\
\hline 0.4376 & 312.53 & 6.61 & 641.20 & 14.30 \\
\hline 0.4374 & 312.34 & 6.71 & 643.19 & 12.74 \\
\hline 0.4372 & 312.31 & 6.62 & 641.28 & 14.08 \\
\hline 0.4352 & 317.60 & 6.57 & 650.11 & 12.59 \\
\hline 0.4349 & 316.90 & 6.55 & 648.41 & 14.08 \\
\hline 0.4354 & 316.61 & 6.57 & 648.36 & 13.48 \\
\hline 0.4359 & 319.73 & 6.56 & 653.83 & 12.55 \\
\hline 0.4354 & 319.76 & 6.54 & 653.40 & 12.49 \\
\hline 0.4350 & 319.79 & 6.55 & 653.93 & 12.21 \\
\hline 0.4360 & 322.69 & 6.60 & 659.95 & 9.09 \\
\hline 0.4355 & 322.60 & 6.57 & 659.32 & 9.31 \\
\hline 0.4363 & 322.74 & 6.58 & 659.58 & 9.59 \\
\hline 0.4359 & 325.50 & 6.52 & 661.43 & 9.54 \\
\hline 0.4362 & 325.78 & 6.51 & 661.40 & 9.70 \\
\hline 0.4357 & 325.74 & 6.51 & 661.34 & 9.42 \\
\hline 0.4353 & 328.55 & 6.48 & 666.22 & 8.75 \\
\hline 0.4356 & 328.48 & 6.45 & 665.05 & 8.27 \\
\hline 0.4342 & 328.61 & 6.49 & 666.84 & 7.99 \\
\hline 0.4340 & 331.61 & 6.48 & 672.04 & 7.17 \\
\hline 0.4341 & 331.56 & 6.46 & 671.43 & 7.30 \\
\hline 0.4342 & 331.58 & 6.47 & 671.71 & 7.11 \\
\hline & & & & \\
\hline
\end{tabular}


Table S14. Experimental conditions and results for mixture 5 (inert) at compressed gas pressure of 6.5 bar and low compression configuration

\begin{tabular}{|c|c|c|c|}
\hline $\mathrm{P}_{\mathrm{i}}($ bar $)$ & $\mathrm{T}_{\mathrm{i}}(\mathrm{K})$ & $\mathrm{P}_{\mathrm{c}}($ bar $)$ & $\mathrm{T}_{\mathrm{c}}(\mathrm{K})$ \\
\hline 0.4157 & 296.75 & 6.47 & 617.95 \\
\hline 0.4152 & 296.67 & 6.45 & 617.41 \\
\hline 0.4150 & 299.35 & 6.43 & 621.83 \\
\hline 0.4163 & 299.20 & 6.44 & 621.56 \\
\hline 0.4209 & 302.69 & 6.51 & 627.87 \\
\hline 0.4218 & 302.59 & 6.53 & 627.67 \\
\hline 0.4313 & 305.01 & 6.67 & 632.03 \\
\hline 0.4314 & 304.78 & 6.67 & 631.63 \\
\hline 0.4365 & 308.47 & 6.68 & 637.00 \\
\hline 0.4366 & 308.27 & 6.71 & 637.48 \\
\hline 0.4370 & 311.05 & 6.68 & 641.62 \\
\hline 0.4375 & 310.74 & 6.69 & 641.07 \\
\hline 0.4373 & 314.16 & 6.65 & 646.75 \\
\hline 0.4370 & 313.78 & 6.76 & 648.63 \\
\hline 0.4351 & 316.94 & 6.71 & 654.28 \\
\hline 0.4348 & 316.79 & 6.71 & 654.01 \\
\hline 0.4348 & 320.73 & 6.67 & 660.18 \\
\hline 0.4349 & 320.24 & 6.71 & 660.17 \\
\hline 0.4350 & 323.40 & 6.66 & 664.94 \\
\hline 0.4354 & 322.27 & 6.68 & 662.93 \\
\hline 0.4357 & 326.37 & 6.64 & 669.77 \\
\hline 0.4357 & 325.78 & 6.66 & 669.16 \\
\hline 0.4352 & 328.84 & 6.69 & 675.46 \\
\hline 0.4350 & 328.70 & 6.68 & 675.22 \\
\hline 0.4347 & 332.83 & 6.60 & 680.76 \\
\hline 0.4344 & 332.39 & 6.62 & 680.42 \\
\hline 0.4349 & 334.60 & 6.58 & 683.45 \\
\hline 0.4350 & 334.39 & 6.60 & 683.53 \\
\hline & & & \\
\hline
\end{tabular}


Table S15. Experimental conditions and results for mixture 3 at compressed gas pressure of 6.5 bar and high compression configuration

\begin{tabular}{|c|c|c|c|c|c|}
\hline $\mathrm{P}_{\mathrm{i}}(\mathrm{bar})$ & $\mathrm{T}_{\mathrm{i}}(\mathrm{K})$ & $\mathrm{P}_{\mathrm{c}}(\mathrm{bar})$ & $\mathrm{T}_{\mathrm{c}}(\mathrm{K})$ & $\tau(\mathrm{ms})$ & $\begin{array}{c}\text { First stage ignition } \\
\text { delay }(\mathrm{ms})\end{array}$ \\
\hline 0.2914 & 297.32 & 6.46 & 655.38 & 26.72 & 12.97 \\
\hline 0.2913 & 297.46 & 6.43 & 655.27 & 27.38 & 10.71 \\
\hline 0.2910 & 297.55 & 6.43 & 655.44 & 25.02 & 12.32 \\
\hline 0.2920 & 305.36 & 6.42 & 669.86 & 18.46 & 4.87 \\
\hline 0.2927 & 305.64 & 6.40 & 669.60 & 18.57 & 4.97 \\
\hline 0.2927 & 305.53 & 6.42 & 669.80 & 20.02 & 5.54 \\
\hline 0.2924 & 303.30 & 6.41 & 665.57 & 19.51 & 7.83 \\
\hline 0.2924 & 303.07 & 6.42 & 665.14 & 21.64 & 7.29 \\
\hline 0.2921 & 302.96 & 6.44 & 665.72 & 22.71 & 7.96 \\
\hline 0.2946 & 309.46 & 6.41 & 676.42 & 15.99 & 4.68 \\
\hline 0.2945 & 309.18 & 6.38 & 675.08 & 15.47 & 5.17 \\
\hline 0.2959 & 308.83 & 6.45 & 675.63 & 15.22 & 5.36 \\
\hline 0.2971 & 312.71 & 6.40 & 681.18 & 12.32 & 3.58 \\
\hline 0.2957 & 312.43 & 6.41 & 681.48 & 14.56 & 3.27 \\
\hline 0.2975 & 312.47 & 6.47 & 681.95 & 14.86 & 2.44 \\
\hline 0.2992 & 317.83 & 6.43 & 690.78 & 12.16 & 2.08 \\
\hline 0.2981 & 318.33 & 6.41 & 691.70 & 14.22 & 1.69 \\
\hline 0.2986 & 317.62 & 6.46 & 691.20 & 13.63 & 2.06 \\
\hline 0.3001 & 322.78 & 6.49 & 701.25 & 12.80 & 1.19 \\
\hline 0.2997 & 323.63 & 6.46 & 702.41 & 13.56 & 1.04 \\
\hline 0.3008 & 323.21 & 6.57 & 703.69 & 14.09 & 1.32 \\
\hline 0.3016 & 327.34 & 6.62 & 712.61 & 17.33 & 0.84 \\
\hline 0.3007 & 326.07 & 6.65 & 711.45 & 17.43 & 1.07 \\
\hline 0.3004 & 326.71 & 6.65 & 712.66 & 16.40 & 0.96 \\
\hline
\end{tabular}

